\title{
La pediatría hospitalaria: futuro desde nuestra historia
}

\author{
OSVALDO ARTAZA B. ${ }^{1}$ \\ 1. Pediatra. Director Hospital Luis Calvo Mackenna. Magíster en Administración. Profesor Agregado Escuela de Salud Publica. \\ Docente Instituto Administración en Salud, Facultad Economía y Negocios de la Universidad de Chile.
}

El presente número de la revista de pediatría nos regala un memorable artículo, publicado el año $1938^{1}$, del doctor Arturo Baeza Goñi, quien fuera presidente de la Sociedad Chilena de Pediatría, y ejerciera a partir de 1921 en el hospital Arriarán, generando múltiples aportes al desarrollo de la pediatría social en nuestro país ${ }^{2}$. Su lectura, así como los pertinentes comentarios de la doctora Schonhaut, son una invitación imposible de declinar a pensar en el futuro de la pediatría hospitalaria justamente desde nuestra historia.

La salud, bien esencial inherente a la dignidad del ser humano, por tanto un derecho, determinante de la calidad de vida y palanca fundamental para el desarrollo de los individuos y las naciones, es resultado de los cambios sociales, políticos, económicos y culturales. Por tanto, a la vez un bien y una construcción social. Es el desarrollo económico ${ }^{3}$, las políticas sociales en su conjunto (saneamiento ambiental, educación, etcétera), junto a políticas públicas en salud persistentes (en control de niño sano, vacunaciones; desnutrición) y sistemas de prestación de servicios basados en los avances científicos y en una atención de primer nivel de amplia cobertura, los que explican nuestros éxitos en la notable disminución de la mortalidad infantil.

Pero dichos éxitos innegables, no han sido iguales para todos. Nuestro sistema de salud aún, a pesar de los avances, se caracteriza por la inequidad ${ }^{4}$ y sigue reflejando un tipo de so- ciedad injusta. La exclusión social es la característica de nuestro modelo de desarrollo ${ }^{5}$. A pesar de la disminución de la pobreza en las últimas décadas, la desigualdad se mantiene, siendo nuestro país -junto a los demás países latino americanos- quienes tienen el indicador de desigualdad (índice de Gini) más elevado del mundo y superior a los que exhiben África y Asia. Ser la región y un país de tan profundas diferencias en el acceso a los resultados del desarrollo explica que no todos los niños y niñas de nuestro país tengan igual oportunidad de vivir y de acceder a los servicios. Hay una parte de Chile que está en la pediatría del mañana y otra que está más cerca de lo que tan bien nos relata el doctor Baeza Goñi. Sin duda que un desafío en Chile para la pediatría social del siglo XXI será contribuir a dar las mismas condiciones y oportunidades a las próximas generaciones.

Amartya Sen, economista de India, premio nobel el año 1998, señaló que el pre requisito esencial para el desarrollo del ser humano es la capacidad de ejercer plenamente la libertad frente a un mundo que ofrece infinitas posibilidades $^{6}$. La pregunta que se hacía el doctor Baeza Goñi y que debemos hacernos nosotros es si niñas y niños podrán en Chile ejercer con igualdad esa opción, o por el contrario se agudizará la crisis de expectativas, crecerá la "indignación" y las consecuencias de un desarrollo humano inequitativo en las futuras generaciones. 
Los éxitos, los "angelitos salvados", nos generan de manera creciente un gran desafío en términos de cronicidad, discapacidad y efectos sobre calidad de vida. Los sobrevivientes de la prematuridad con sus secuelas neurológicas, oftalmológicas, digestivas y pulmonares; los sobrevivientes de la cirugía cardíaca y sus secuelas de largo plazo; la sobrevida del cáncer infantil; las secuelas de las correcciones del aparato urogenital; la creciente sobrevida de la fibrosis quística y un largo etcétera, que plantean a la pediatría del mañana no sólo interrogantes sino que sobre todo responsabilidades de las cuales hacerse cargo.

Junto a lo anterior, la pediatría del mañana tiene que responder a los "males del desarro1lo", la obesidad, el maltrato y el abuso infantil, el fenómeno del bullying en la escuela, y una larga lista de nuevos hechos y fenómenos de una "infancia infeliz" en que la niña y el niño tienen que desarrollarse en el contexto de la diversidad de "ser familia" hoy; de la disfuncionalidad de las relaciones del mundo adulto; de la soledad del padre muchas veces ausente y la madre que debe ejercer esa doble presencia: la laboral y la de jefa de hogar; de la maternidad a edades extremas, embarazo adolescente o el tardío de la mujer que pospuso ser madre luego de alcanzar un estatus profesional, de la violencia de los grupos excluidos que buscan a su manera su espacio en la sociedad, de la violencia de las elites en sus intentos de mantener el status quo, de la violencia a resultas de la droga, de la violencia como consecuencia de grupos humanos que han perdido el sentido. Nosotros nos enfrentamos a la mal nutrición por exceso, a los "trastornos de la modernidad", a las dificultades de niña y niños para adaptarse al modelo instructivo de la escuela actual. Nosotros -los pediatras de hoy y del mañana- igual que el doctor Baeza Goñi, seguiremos luchando cual quijotes con "los molinos de viento". La salud de las niñas y de los niños en mucho es y será reflejo del tipo de sociedad que somos.

El contexto hoy es de un mundo en constantes, profundos, inevitables y más veloces $\operatorname{cambios}^{8}$, de la mano de incrementos exponenciales de conocimiento, incertidumbre, complejidad, globalidad e interconexiones. Este contexto explica grandes tendencias: cambios de las poblaciones (migraciones, urbanización creciente), los tipos de familia y de los problemas de salud de los niños; incremento de los avances científicos y de las tecnologías de la información; modificaciones constantes en los sistemas de salud; nuevos paradigmas y comportamientos de los usuarios de los sistemas (patrones culturales que se modifican generando mayor diversidad, fragilidad de la asociatividad -tejido social- convencional, en paralelo a mayor conectividad tecnológica y empoderamiento de derechos en sociedad de consumo "tiempos de soledad interconectada"), entre muchos otros cambios que afectan nuestra práctica cotidiana.

Los problemas de salud complejos y crónicos de la infancia, con padres más informados y empoderados (de la familia tradicional, más extensa, a una familia muchas veces con un solo progenitor, o donde ambos trabajan fuera del hogar con el incremento de niños en salas cunas. Donde cada vez nacerán menos niños -que serán paradójicamente más cuidados y mimados, a la vez que serán puestos -culposamente- al cuidado de terceros con mayor frecuencia-) que no aceptan la muerte (perplejidad y no aceptación ya que se ve a la ciencia como capaz de todo y cualquier fallo como una negligencia del equipo de salud), tendremos que enfrentarlos cada vez con más dificultad para tener los recursos (financieros, humanos y tecnológicos), ya que la competencia por ellos desde los infinitos requerimientos de la medicina del adulto mayor será cada vez más dura.

Ante esta dura batalla por los recursos, tendremos que afinar argumentos con respecto al impacto en la competitividad país de una fuerza de trabajo que ve afectada su desempeño por las enfermedades crónicas de sus hijos; por los desordenes neurológicos que afectan su escolaridad; por el riesgo de trauma y adicciones en la adolescencia, entre otros. Ya hay estudios que dan sólidos argumentos en que invertir en la infancia y la adolescencia, junto a la ganancia en años de vida saludables, tiene su rédito en incrementos de productividad ${ }^{9,10}$.

Parte importante de este debate con respecto al aporte de la pediatría al desarrollo humano en nuestro país tendrá que ver con la defi- 
nición -que urge tomen nuestras autoridadessobre el ingreso formal de la adolescencia en la práctica hospitalaria pediátrica. Ingreso que deberá ser necesariamente gradual por las particulares exigencias que los adolescentes nos ponen en términos de nuestras competencias e infraestructura.

Así como un mundo en rápido cambio nos señala sus tendencias, la práctica pediátrica ha tenido que ir adaptándose rápidamente. Dichas tendencias podemos resumirlas en:

La práctica seguirá exigiéndonos privilegiar la atención ambulatoria y evitar hospitalizaciones innecesarias, junto a comprender que ya no caben miradas individuales o de compartimentos, cada vez más la atención pediátrica tendrá mayor base sistémica. El equipo de salud pediátrico es crecientemente multi e interprofesional ya que son variadas y diversas las competencias para resolver y acompañar las necesidades de salud del niño y del adolescente. La práctica ya no está centrada en el "paciente" sino en la ("las" dada la diversidad) familia, ya que el o los padres y/o tutores exigen no sólo información, sino que también participación en las decisiones a la vez que requieren de apoyo y manejo multi disciplinario ${ }^{11,12}$. La práctica ya no está centrada en un órgano enfermo sino en procesos asistenciales que no empiezan ni terminan en un centro de salud, sino que integran distintos dispositivos -atención de primer nivel, secundaria y terciaria, atención ambulatoria y de internación- a través de protocolos y guías co-construidos desde la necesidad del niño, su familia y medio. Integración en multi dimensión: la espacial -diversos dispositivos-; la temporal -en el ciclo de vida- y la biopsicosocial -en la complejidad de lo humano- ${ }^{13}$.

Estas nuevas prácticas requieren nuevas competencias para el pediatra, las que deben estar presentes en todo su proceso de aprendizaje continuo. Competencias para conversaciones efectivas en un contexto de creciente interdependencia y de equipos más amplios e inter disciplinarios dentro y fuera de los centros de salud, habilidades para trabajar con el niño y el adolescente en un contexto de familia y medio, usando las distintas tecnologías de la información; y por sobre todo capacidad de aprender con otros, investigar, innovar, adap- tar continuamente las prácticas, formando parte de comunidades humanas de aprendizaje ${ }^{14}$. La investigación traslacional de avanzada no puede hoy separarse de las nuevas prácticas en pediatría, señalo como ejemplo -no exento de orgullo- las líneas de investigación en insuficiencia renal, trasplantes y neutropenias febriles que hoy se están desarrollando en el Luis Calvo Mackenna, teniendo como soporte a consolidados laboratorios de biología molecular y genética surgidos de visionarios convenios entre el hospital y la Universidad de Chile.

Estas nuevas prácticas requieren también de un nuevo tipo de hospital, de estructura y arquitectura abierta y flexible, que comprende y refuerza el rol central de la atención de primer nivel, que forma parte de sistemas integrados, que al centro de su quehacer coloca las necesidades y los derechos del niño, y que genera contextos para la innovación, el aprendizaje y la calidad de vida de quienes allí trabajan. No se podrán dar cuidados efectivos a niños gravemente enfermos o con dolencias crónicas desgastantes para sus familias y para sus cuidadores sin gran inversión de energía en la calidad de vida de quienes trabajamos dando soporte a dichos niños en contexto de permanente escasez de recursos financieros (nunca de imaginación).

Estas nuevas prácticas requieren de un nuevo tipo de Universidad y porque no decirlo de sociedad. De una Universidad que se pregunte sobre el lugar donde se debe formar al pediatra para las necesidades de hoy y del mañana y sobre sus competencias ya no sólo técnicas, sino fundamentalmente relacionales y adaptativas para el aprendizaje y la interacción en un mundo cambiante que exige de miradas sistémicas ${ }^{15}$. Que se pregunte sobre si el médico se puede formar separado de las demás profesiones de la salud y de las ciencias sociales, o si en cambio, las mallas tendrán que ser más transversales y compartidas. ¿Cómo pedir trabajo en equipo si no se ha formado en equipos? Una universidad que superando la mera instrucción, habla de valores y de sueños y debate sobre herramientas para la innovación en un mundo complejo ${ }^{16}$.

Finalmente estas nuevas prácticas nos obli- 
gan a mirar nuestro modelo de sociedad y las consecuencias que un mercado insuficientemente regulado (teniendo un rol tan preponderante en las políticas sociales, en un país con tan escasa capacidad para distribuir de manera justa y equitativa el fruto del desarrollo), tiene sobre nuestros niños y adolescentes.

Al final, parafraseando a Virchow, pediatría es una ciencia social y las políticas no es otra cosa que medicina a gran escala ${ }^{17}$. El reclamo de los niños explica que los pediatras de ayer fueran protagonistas en desarrollar programas y políticas exitosas para enfrentar la mal nutrición y los problemas infecto contagiosos. El reclamo de los niños de hoy nos pide el mismo liderazgo y maestría para enfrentar ya no los desafíos de la sobrevida, sino las exigencias de una vida plena, el llamado del florecimiento de lo humano. Detrás del pediatra de ayer, de hoy y del mañana una misma capacidad de soñar y de creer en nuevos mundos posibles para los niños y niñas de Chile.

\section{Referencias}

1.- Baeza A: Estadística del Servicio de Lactantes del Hospital Arriarán: Ensayo de clasificación. Publicado en Rev Chil Ped 1938; 1000-4.

2.- Laval E: Recuerdo histórico del antiguo Servicio de Infecciosos del Hospital de Niños Manuel Arriarán. Rev Chil Infect 2008; 25 (4): 301-6.

3.- Kjellmer I: The paediatrician -past, present and future- a conversation with Nils Rosén von Rosentein Acta Paediatrica 2009; 98: 1392-99.

4.- WHO Commission on Social Determinants of Health 2008: Closing the gap in a generation. Health equity through action on the social determinants of health. Disponible en http://www.who.int/social_determinants/ thecommision/finalreport/en/

5.- Artaza O: Reformas y Financiamiento de Sistemas de Salud de la Subregión Andina. Organismo Andino de Salud Lima Perú. Págs. 7-16.

6.- Sen A: Development as freedom. Oxford University Press. 1999.

7.- Jiménez J: Angelitos Salvados. Ugbar editores. Chile 2009; Págs. 15-31.

8.- Leslie L, et al: Peering into the future: Pediatrics in a changing world. Pediatrics 2010; 126 (5): 982-8.

9.- Fluet $P$, et al: Benefits for employees with children with special needs: findings from the collaborative employee benefit study. Health Aff. 2007; 26: 1096-103.

10.- Heckman $J$, et al: Investing in our Young people. In: Childhood Programs and Practice in the First decade of Life. New York. Cambridge University Press; 2010.

11.- Zolnierek $H$ : Phisician communication and patient adherence on treatment: a meta analysis. Med Care 2009; 47: 826-34.

12.- Kuo D, et al: Family-Centered care: Current Applications and Future Directions in Pediatric Health Care. Matern Child Health J 2012; 16: 297-305.

13.- OPS: Redes Integradas de Servicios de Salud: el desafío de los hospitales. Chile 2011 Págs. 59-136.

14.- Artaza O: El hospital público como comunidad humana de aprendizaje. Igd Ed. Chile 2010 Págs. 53-56.

15.- Perrin J, Dewitt T: Future of Academic General Pediatrics - Areas of Opportunity. Academic Pediatrics 2011; V 11, 3: 181-8.

16.- Frenk $J$, et al: Professionals for as new century: transforming education to strengthen health systems in an interdependent world. The Lancet 2010; 376: 1923 58.

17.- Eisemberg L, Virchow L: Where are you now that we need you? Am J Med 1984; 77: 524-32. 